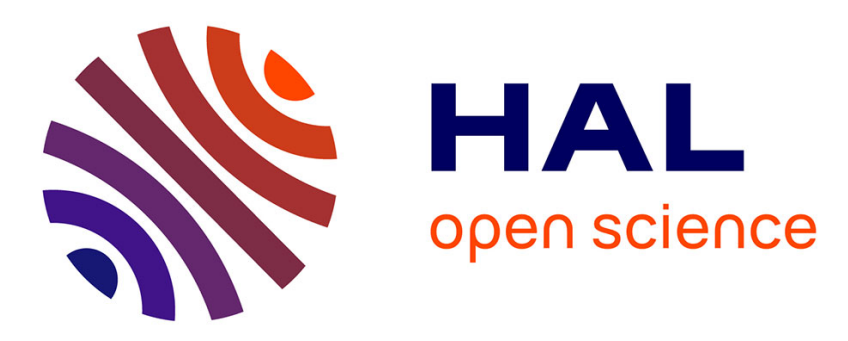

\title{
Application of principal component analysis on NIR spectral collection after elimination of interference by a least-squares procedure
}

\author{
M.F. Devaux, D. Bertrand, P. Robert, M. Qannari
}

\section{- To cite this version:}

M.F. Devaux, D. Bertrand, P. Robert, M. Qannari. Application of principal component analysis on NIR spectral collection after elimination of interference by a least-squares procedure. Applied Spectroscopy, 1988, 42 (6), pp.1020-1023. hal-02727128

\author{
HAL Id: hal-02727128 \\ https://hal.inrae.fr/hal-02727128
}

Submitted on 2 Jun 2020

HAL is a multi-disciplinary open access archive for the deposit and dissemination of scientific research documents, whether they are published or not. The documents may come from teaching and research institutions in France or abroad, or from public or private research centers.
L'archive ouverte pluridisciplinaire HAL, est destinée au dépôt et à la diffusion de documents scientifiques de niveau recherche, publiés ou non, émanant des établissements d'enseignement et de recherche français ou étrangers, des laboratoires publics ou privés. 


\title{
Application of Principal Component Analysis on NIR Spectral Collection after Elimination of Interference by a Least-Squares Procedure
}

\author{
M. F. DEVAUX, D. BERTRAND, ${ }^{*}$ P. ROBERT, and M. QANNARI \\ Institut National de la Recherche Agronomique, Laboratoire de Technologie Appliquée à la Nutrition (M.F.D., D.B., P.R.) and \\ Ecole Nationale d'Ingénieurs des Techniques des Industries Agricoles et Alimentaires, Chaire de Mathématique (M.Q.), Rue \\ de la Géraudière, 44072 Nantes, Cedex 03, France
}

In NIR spectroscopy, multidimensional analyses such as Principal Component Analysis (PCA) may be applied to examine the similarity between spectra of natural products. However, such an approach is often limited by the effect of spectral interference due to water or particle size distribution of the samples. In the present work, the advantage of the elimination of such spectral interference before performing PCA was investigated. Unwanted component spectra were eliminated by a least-squares procedure. They were first orthogonalized and normalized by the GramSchmidt orthogonalization method. The subtraction coefficients were then assessed, similarly to principal component (PC) scores, by projection of the NIR spectra on the orthogonalized component spectra, and PCA was performed on the corrected spectra. This method was applied on an illustrative collection of wheat semolina conditioned in three levels of water content. Water was the component to be eliminated and had been previously modeled by two spectral patterns. These spectral patterns were used as the unwanted component spectra. PCA was applied independently before and after spectral correction of the collection of spectra and graphs obtained by the two procedures were compared. The squared correlation coefficient of the 3 first PC scores with water content was 0.979 before correction, with the 3 groups of water content appearing clearly on PCA graphs. After correction, the corresponding squared correlation coefficient for the 7 first PC scores was 0.016. PCA graphs obtained with corrected spectra also showed that the water effect was completely eliminated. At this moment, samples were separated according to their technological nature. The procedure developed may be useful in pattern recognition study and for automatic clustering of NIR spectra. It may also be applied in fields other than NIR spectroscopy.

Index Headings: Infrared; Analytical methods; Reflectance spectroscopy; Spectral deconvolution; Elimination of interferences.

\section{INTRODUCTION}

Near-Infrared Reflectance Spectroscopy (NIRS) is now widely applied in order to predict the composition of various food products. ${ }^{1}$ Generally, prediction equations are established by multilinear regression between spectral absorbances and known biochemical values of samples. Multidimensional analyses are another way of extracting useful information from spectra. Bertrand et al. ${ }^{2}$ and Devaux et al. ${ }^{3}$ have shown that Principal Component Analysis (PCA) is a means of identifying wheats according to their variety or baking quality. Robert et al. ${ }^{4}$ have applied PCA to separate forage samples according to their in vitro digestibility. Cowe and $\mathrm{McNicol}^{5}$ have shown that PCA splits up a spectrum into a sum of orthogonal spectral patterns. PCA gives graphical representations of collections where similar spectra are neighboring. However, the criteria of similarity are somewhat arbi-

Received 16 November 1987; revision received 2 March 1988.

* Author to whom correspondence should be sent. trary because they are linked to particle size and water content, which are the most variable phenomena in the NIR spectra. ${ }^{6}$ Two samples of similar biochemical composition but of different particle size and water content will be far from each other on the PCA graphs. This separation is generally irrelevant. For instance, in the wheat industry, the water content of flours is not a good criterion for qualitative technological classification. It would be useful to eliminate the irrelevant spectral components, such as that of water, in each spectrum before application of PCA. In Middle-Infrared Analysis (MIA), this problem is solved by subtracting the unwanted spectral component according to:

$$
s_{c}=s-k \times \mathbf{p}
$$

where $s$ is the sample spectrum, $\mathbf{p}$ is the spectral component of the unwanted phenomenon, $k$ is the subtracting coefficient, and $s_{c}$ is the corrected spectrum. This procedure requires the knowledge of $\mathbf{p}$, and the determination of $k$. It is common for the value of $k$ to be graphically assessed by a skilled operator. This procedure is time-consuming and cannot be applied in NIR spectroscopy, because spectra of food products reveal no sharp peaks. Other methods have been advanced to assess the subtraction coefficient in order to obtain a reproducible subtraction; ${ }^{7}$ most of them are based on leastsquares curve-fitting. ${ }^{8,9}$ These methods often assume that there is an area of the spectrum where only the components subtracted have absorption bands. Such an hypothesis cannot be made in NIRS, because all the usual food components have overlapping bands.

When there are no interactions between the biochemical components, $\mathbf{p}$ is the spectrum of the pure unwanted component. However, p may be difficult to record separately, because the pure component is not always available. Moreover, the pure component spectrum may be different from the corresponding spectrum in the mixture. Methods have been advanced for assessing component spectra on the basis of the correlations between biochemical values and spectral values. ${ }^{10,11}$

In a previous study ${ }^{12}$ component spectra were assessed by the application of multidimensional analyses. Factorial Discriminant Analysis (FDA) was performed on $a$ priori groups of spectra and enabled discriminant spectral patterns to be extracted. In the present study, a leastsquares method has been used to assess the subtracting coefficients $k_{i}, i=1,2 \ldots m$, assuming that the undesired component spectra $\mathbf{p}_{i}, i=1,2 \ldots m$ are known. The method permits the automatic subtraction of several 


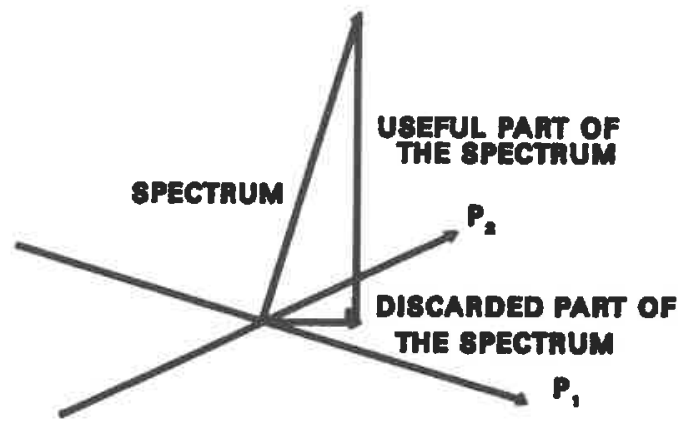

FIG. 1. Principle of elimination of spectral interference.

component spectra and is consistent with the logic of PCA. The purpose of the present work was to study the potential value of PCA-based pattern recognition on data which have been preprocessed to reduce interference. The work has been done on an illustrative collection of wheat products differing in their water content.

\section{THEORY}

Principal Component Analysis. PCA $^{13}$ has been described in a previous study. ${ }^{12}$ Only the main characteristics are reported here. PCA is a method of description and compression of large data sets. PCA provides a vector representation of spectral data: each spectrum can be seen as a point in a $v$ dimensional space where $v$ is the number of wavelengths. The aim of PCA is to find another system of orthogonal axes where the variances of the collection of spectra is maximum on the first axes. Generally, the absorbances at different wavelengths are highly correlated and much fewer axes than $v$ are necessary to describe almost all the variance. The projection of the spectra on the new axes gives factorial coordinates called "PC scores." The solution is to assess the eigenvectors of the variance-covariance matrix of the spectral data. Then the PC scores are assessed by the projection of the spectral data on these eigenvectors

$$
\mathbf{C}=\mathbf{D U}^{\mathrm{T}}
$$

where $\mathbf{C}$ is the $n \times a$ matrix of PC scores, $\mathrm{U}$ is the $a \times$ $v$ matrix of the eigenvectors, $\mathbf{U}^{\mathrm{T}}$ is the transposed matrix of $\mathbf{U}, \mathbf{D}$ is the $n \times v$ matrix of the spectral data, $n$ is the number of samples, $v$ is the number of wavelengths, and $a$ is the number of significative PC scores. Generally, 15 axes are sufficient for the description of NIR spectral data of natural products. Graphs of two chosen PC scores may be drawn. They are significant for the similarity between the samples.

It has been shown ${ }^{5,12}$ that PCA carries out a spectral decomposition:

$$
s=c_{1} \mathbf{u}_{1}+c_{2} \mathbf{u}_{2}+\ldots
$$

where $c_{1}, c_{2} \ldots$ are the $\mathrm{PC}$ scores corresponding to the spectrum $s$, and $\mathbf{u}_{1}, \mathbf{u}_{2}, \ldots$ are the eigenvectors from matrix $\mathbf{U}$.

\section{ELIMINATION OF UNWANTED COMPONENT SPECTRA}

Component spectra have been eliminated by a leastsquares procedure. When there are several component spectra, $s$ can be written from Eq. 1:

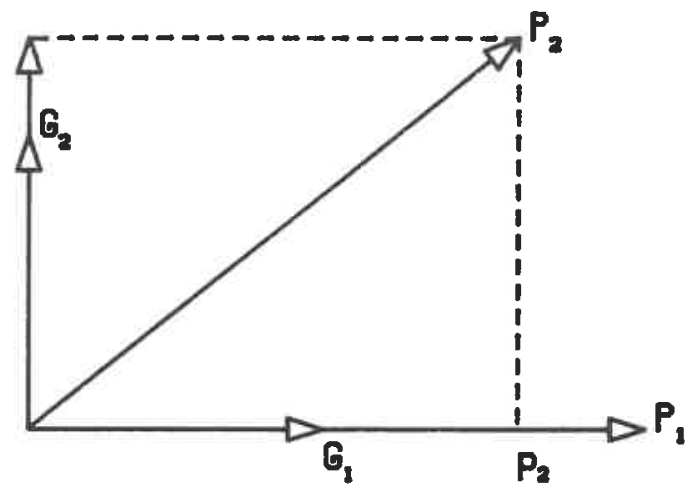

FIG. 2. Principle of the Gram-Schmidt orthogonalization method.

$$
s=s_{c}+k_{1} \mathbf{p}_{1}+k_{2} \mathbf{p}_{2}+\ldots
$$

If PCA is performed on the collection of the corrected spectra $s_{v}, s_{c}$ is decomposed according to Eq. 3:

$$
s_{c}=a_{1} \mathbf{v}_{1}+a_{2} \mathbf{v}_{2}+\ldots
$$

where $a_{1}, a_{2} \ldots$ are the PC scores of the corrected spectra $s_{e}$, and $\mathbf{v}_{1}, \mathbf{v}_{2} \ldots$ are the PCA eigenvectors. From Eqs. 4 and 5:

$$
s=a_{1} \mathbf{v}_{1}+a_{2} \mathbf{v}_{2}+\ldots+k_{1} \mathbf{p}_{1}+k_{2} \mathbf{p}_{2}+\ldots
$$

$s$ is then split up into two parts: $k_{1} \mathbf{p}_{1}+k_{2} \mathbf{p}_{2}+\ldots$, corresponding to the unwanted part, and $a_{1} \mathbf{v}_{1}+a_{2} \mathbf{v}_{2}+$ ..., corresponding to the relevant part. Figure 1 illustrates this decomposition. $\mathbf{p}_{1}, \mathbf{p}_{2}, \ldots$ form a subspace where the undesired part of the spectrum is projected. The useful part is obtained by vector subtraction of the discarded part from the initial spectrum. The coefficients $k_{i}$ are in fact the regression coefficients given by the wellknown least-squares method. However to be consistent with PCA, it is logical to give to $\mathbf{p}_{1}, \mathbf{p}_{2}, \ldots$ the same properties as those of the PCA eigenvectors $\mathbf{v}_{1}, \mathbf{v}_{2}, \ldots$ : the $\mathbf{p}_{\mathbf{i}}$ vectors must be unit vectors and orthogonal to each other. The subtracting coefficients $k_{i}$ are calculated similarly to the PC scores $a_{i}$ by projection of the spectra

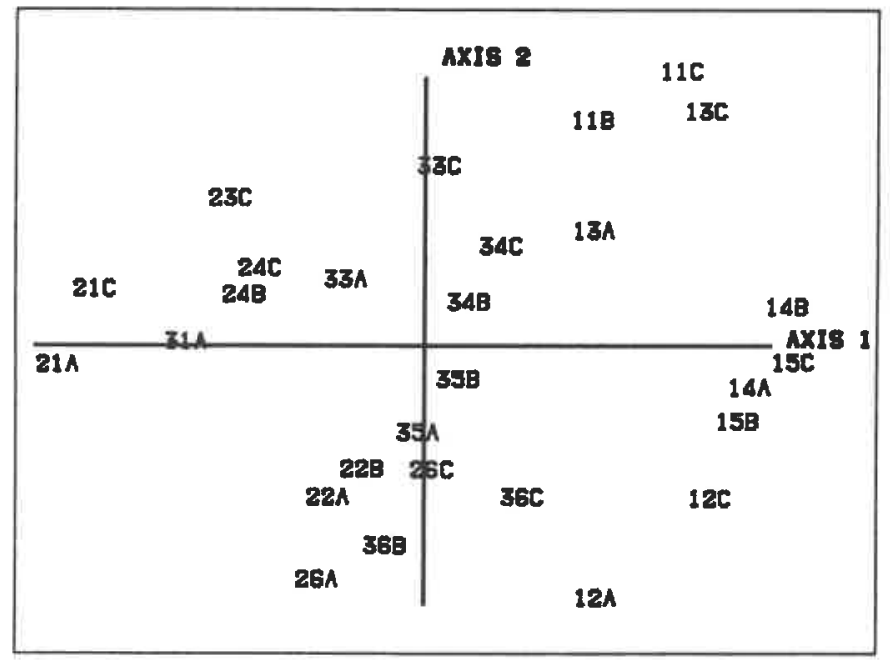

FIG. 3. Representation of PC scores 1 and 2 without elimination of the effect of water. Codes: 1st letter: 1, 2, and 3 for the three wheats; 2nd letter: $1,2,3,4,5$, and 6 for the six technological natures of the semolinas; 3rd letter: A, B, and C for the three groups of water content. Coincident samples: $23 \mathrm{~B}$ with $23 \mathrm{C}$; $31 \mathrm{C}$ with $24 \mathrm{~B}$. 


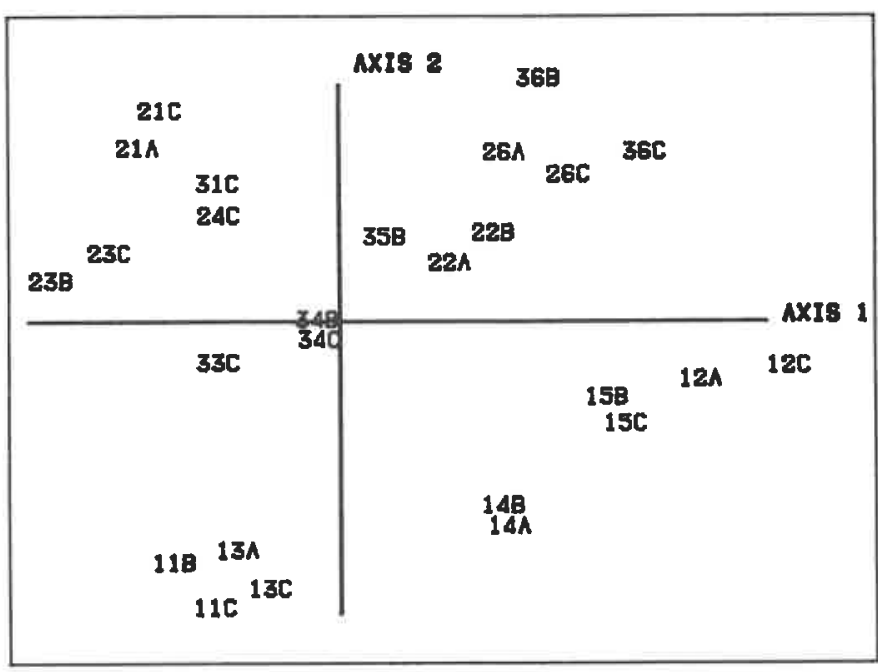

FIG. 4. Representation of PC scores 1 and 2 after elimination of the effect of water. Codes: same as in Fig. 3. Coincident samples: 31A with $24 \mathrm{C}$; $24 \mathrm{~B}$ with $24 \mathrm{C}$; $33 \mathrm{~A}$ with $33 \mathrm{C}$; $35 \mathrm{~A}$ with $35 \mathrm{~B}$.

on orthogonal vectors. Generally, $\mathbf{p}_{\mathbf{i}}, \mathbf{p}_{2}, \ldots$ are not orthogonal and not unit vectors. It is then necessary to orthogonalize them. This has been done by the GramSchmidt orthogonalization method. The orthogonalization aids in conditioning the correlation matrix of $\mathbf{p}_{\mathrm{i}}$ vectors for inversion.

\section{GRAM-SCHMIDT ORTHOGONALIZATION METHOD}

The Gram-Schmidt orthogonalization method has been previously applied in the middle-infrared region by different authors. ${ }^{14,15}$ The principle of the method is illustrated in Fig. 2 for two-component spectra. A first component spectrum $\mathbf{p}_{1}$ is normalized according to:

$$
\mathbf{g}_{1}=\frac{\mathbf{p}_{1}}{\left\|\mathbf{p}_{1}\right\|}
$$

where $\left\|\mathbf{p}_{1}\right\|$ is the norm of $\mathbf{p}_{1}$. The projection of the second component $\mathbf{p}_{2}$ on $\mathbf{g}_{\mathbf{1}}$ is assessed by the scalar product:

$$
\mathbf{p}_{12}=\mathbf{p}_{2} \mathbf{g}_{1}{ }^{\mathrm{T}} \text {. }
$$

The orthogonal part of $\mathbf{p}_{2}$ is given by:

$$
\mathbf{p}_{2}-\mathbf{p}_{12} \mathbf{g}_{1} \text {. }
$$

$\mathbf{g}_{2}$ is then calculated by the normalization of $\mathbf{p}_{2}-\mathbf{p}_{12} \mathbf{g}_{1}$.

When there are more than two component spectra, the process is iterated: the spectrum $\mathbf{p}_{3}$ is orthogonalized with $\mathrm{g}_{1}$, similarly to Eqs. 8 and 9, and the result is orthogonalized with $\mathrm{g}_{2}$ in a same manner and finally normalized, giving $\mathbf{g}_{3}$. The procedure can be applied with any number of component spectra $\mathbf{p}_{\mathbf{i}}$.

\section{CALCULATION OF THE SUBTRACTION COEFFICIENTS}

The subtraction coefficients are calculated by orthogonal projection of the spectra on the $\mathrm{g}$ vectors according to:

$$
\mathbf{K}=\mathbf{S G}^{\mathbf{T}}
$$

where $\mathbf{K}$ is the $n \times m$ matrix of the subtraction coeffi- cients, $\mathbf{S}$ is the $n \times v$ spectral data matrix, $\mathbf{G}^{\mathrm{T}}$ is the $v$ $\times m$ transposed matrix of the $\mathbf{g}$ vectors, $m$ is the number of undesired component spectra, $n$ is the number of spectra, and $v$ is the number of wavelengths.

\section{CALCULATION OF THE CORRECTED SPECTRA}

The corrected spectral data matrix $\mathbf{S}_{\mathbf{c}}$ is given by:

$$
\mathbf{S}_{\mathbf{c}}=\mathbf{S}-\mathbf{K G} \text {. }
$$

PCA is then performed on the matrix of the corrected spectra $\mathbf{S}_{\mathbf{c}}$.

\section{EXPERIMENTAL}

The method has been tested on an illustrative collection of semolina of six technological types coming from three wheats and conditioned in three levels of water content. Samples and spectroscopic measurements were described in a previous work. ${ }^{12}$ The aim of the present study was to eliminate the differences between the spectra only due to variation in water content. In the previous work $^{12}$ it was shown that the effect of water on the NIR spectra could be modeled by the sum of two spectral components called "discriminant spectral patterns." These two patterns were chosen as unwanted component spectra in the present work.

All mathematical processing was performed on a minicomputer, PRIME 750. All programs were written at the authors' laboratory. The two discriminant spectral patterns of water were orthogonalized by the Gram-Schmidt method. Then PCA was performed on the spectra. At the same time, classical PCA was achieved on the same spectral data without any pretreatments.

\section{RESULTS AND DISCUSSION}

Principal Component Analysis of the Original Spectral Data. Eigenvectors 1, 2, and 3 were described in the previous study..$^{12}$ The squared correlation coefficients of the three corresponding PC scores with water content were, respectively, $0.017,0.208$, and 0.754 . Thus, the multiple squared correlation coefficient is 0.979 ; almost all the effect of the water is described by the PC scores 2 and 3. The representation of PC scores 1 and 2 (99\% of the total variance) is given in Fig. 3. Axis 1 is significative of the particle size and the texture of the samples. The semolina coming from wheat 1 is clearly separated from the others. This result is probably due to differences of texture. Samples differing only by their water content are rather far apart: see, for example, $26 \mathrm{~A}$ and $26 \mathrm{C}$ or $12 \mathrm{~A}$ and $12 \mathrm{C}$. Moreover, when water content increases (from A to C), samples are always shifted in the same direction, showing the water gradient.

Principal Component Analysis after Elimination of the Effect of Water. For this analysis, the squared correlation coefficients of the three first PC scores with water content were, respectively, $0.004,0.005$, and 0.001 , and the multiple squared correlation coefficient for the seven first PC scores was 0.016 . So the new PC scores have no signification in terms of water content. The representation of PC scores 1 and 2 is given in Fig. 4. Axis 1 mainly 
distributes the samples according to the technological nature of the semolinas. (From the left to the right: semolinas 3 and $1,4,5,2,6$.) This result is roughly in agreement with known technological qualities of the semolina. ${ }^{16}$ Axis 2 now separates the wheat 1 from the other two. Samples only differing by their water content are now neighboring, and the water gradient is no longer visible. The water effect has thus been completely eliminated.

\section{CONCLUSIONS}

On the basis of the studied illustrative collection, the method suggested was efficient in eliminating the unwanted component spectra of water. It is possible to model the effect of a single component by more than one spectrum. This may be a way to take into account nonlinear distortions. By the same procedure, it was possible to eliminate the effect of texture in NIR spectroscopy of wheat products. ${ }^{17}$ The method may be applicable in other fields, such as middle-infrared spectroscopy, chromatography, and electrophoresis, in order to eliminate baseline variations. The procedure is consistent with the logic of multidimensional analyses and defines criteria of similarity more relevant than those of classical PCA. This may be a useful tool for the creation of spectral libraries ${ }^{18}$ or for clustering. ${ }^{19}$
1. B. G. Osborne and T. Fearn, Near Infrared Spectroscopy in Food Analysis, Longman Scientific \& Technical (John Wiley \& Sons, New York, 1986).

2. D. Bertrand, P. Robert, and W. Loisel, J. Sci. Food Agric. 36, 1120 (1985).

3. M. F. Devaux, D. Bertrand, and G. Martin, Cereal Chem. 63, 151 (1986).

4. P. Robert, D. Bertrand, and C. Demarquilly, Anim. Feed Sci. and Technol. 16, 215 (1986).

5. I. A. Cowe and J. W. McNicol, Appl. Spectrosc. 39, 257 (1985).

6. P. C. Williams and B. N. Thompson, Cereal Chem. 55, 1014 (1978).

7. P. C. Gillette and J. L. Koenig, Appl. Spectrosc. 38, 334 (1984).

8. J. R. Powell, F. M. Wasacz, and R. S. Jacobsen, Appl. Spectrosc. 40, 339 (1984).

9. M. K. Antoon, J. H. Koenig, and J. L. Koenig, Appl. Spectrosc. 31, 512 (1977).

10. D. E. Honigs, G. M. Hieftje, and T. Hirschfeld, Appl. Spectrosc. 38, 317 (1984).

11. B. G. Osborne, J Sci. Food Agric. 35, 106 (1984).

12. M. F. Devaux, D. Bertrand, P. Robert, and M. Qannari, Appl. Spectrosc. 42, 1015 (1988).

13. L. Lebart, A. Morineau, and N. Tabard, Techniques de la Description Statistique (Dunod Bordas, Paris, 1977), Chap. 1.

14. C. H. Brown, R. J. Obremski, and P. Anderson, Appl. Spectrosc. 40, 734 (1986).

15. M. R. Nyden, Appl. Spectrosc. 40, 868 (1986).

16. D. Bertrand, P. Robert, M. F. Devaux, and J. Abecassis, Spectroscopy across the Spectrum: Analytical Applications of Spectroscopy, Norwich, UK (1987), Paper number 32.

17. M. F. Devaux, D. Bertrand, P. Robert, and J. L. Morat, Euroanalysis VI, Paris (1987), Paper number Fr 209.

18. Ching Po Wang and T. L. Isenhour, Appl. Spectrose. 41, (1987).

19. T. Naes, Journal of Chemometrics 1, 121 (1987). 
